\title{
OPEN Dose-response effects of light therapy on sleepiness and circadian phase shift in shift workers: a meta-analysis and moderator analysis
}

\author{
Calvin Lam ${ }^{1} \&$ Min-Huey Chung 2,3 的
}

Light therapy has been considered to be effective in mitigating sleepiness and regulating circadian phase shift in shift workers. However, the effective treatment dose of light therapy remains undetermined. We performed a meta-analysis of randomized experimental studies to determine the effect of light therapy doses on sleepiness and circadian phase shift in shift workers. An article search was performed in 10 electronic databases from inception to June 2020. Two raters independently screened and extracted data and reached consensus. Twenty-one eligible studies were included. Analyses were performed using random-effects models. Light therapy exerted significantly small to medium effects on sleepiness and large treatment effects on circadian phase shift. Moderator analyses performed with subgroup and metaregression analyses revealed that medium-intensity light therapy for a shorter duration more effectively reduced sleepiness at night, whereas higher-intensity light therapy more effectively induced phase shifting, but the required treatment duration remained inconclusive. This study provides evidence regarding the effect of light therapy in reducing sleepiness and shifting circadian phase in shift workers. Exposure to medium-intensity light for a short duration at night reduced sleepiness, whereas exposure to high-intensity light improved sleep by shifting their circadian phase.

Shift work involves staff members working during the day, evening, or night, enabling an organization to operate longer than individual workers can work ${ }^{1-3}$. Quick-rotating night shifts may reduce the attentional performance of the shift workers because of the generation of a higher level of prolactin (a sleep-related hormone) in daytime; in addition, fatigue, daytime sleepiness, and insomnia have been found in shift workers ${ }^{4-7}$. Scheduled shift work is required in many industries, such as the nursing sector; therefore, mitigating the sleep problems of shift workers is crucial to sustain their energy and health status.

Sleepiness and circadian phase problems are two critical examples of sleep problems in shift workers. Sleepiness is a condition marked by inactivity and a tendency to fall asleep ${ }^{8,9}$. Reducing the sleepiness of shift workers during shift work can increase work performance and reduce accidents ${ }^{4,10}$. Light therapy is a nonpharmacological treatment that has been considered effective in mitigating the sleepiness of shift workers. The use of light exposure at nighttime to delay the sleep phase may increase alertness for working at night and increase daytime sleepiness ${ }^{11,12}$. By contrast, light exposure during daytime to advance the sleep phase may regulate and increase nighttime sleepiness on days off ${ }^{13}$. After light exposure, shift workers may exhibit improved alertness at work and improved sleep after work and on days off.

The circadian phase problem is a misalignment between the circadian clock and sleep-wake schedule ${ }^{13}$. For instance, individuals have a normal sleep-wake schedule of sleeping at nighttime and wake up in the morning; however, shift workers may work at night, sleep in the morning, and wake up in the afternoon or evening. Shifting the circadian phase to an adaptive state for shift work may reduce sleep problems caused by shift work. Individuals exposed to artificial bright light or sunlight under light therapy have exhibited a circadian phase

${ }^{1}$ Department of Psychology, The University of Hong Kong, Pok Fu Lam, Hong Kong. ${ }^{2}$ School of Nursing, College of Nursing, Taipei Medical University, Taipei, Taiwan. ${ }^{3}$ Department of Nursing, Shuang Ho Hospital, Taipei Medical University, New Taipei City, Taiwan. ${ }^{\circledR}$ email: minhuey300@tmu.edu.tw 
shift ${ }^{13-17}$ because exposure to light may have suppressed melatonin and shifted the sleep phase ${ }^{11,12}$. Hence, light therapy may adjust the timing of melatonin secretion to coincide with the sleep phase.

To effectively use light therapy to enhance the sleep health of shift workers, understanding whether light therapy exerts a dose-response effect on the sleep problems of shift workers is crucial. However, this is a debated topic. The dose of light therapy is measured on the basis of light intensity (lux) and treatment duration, expressed as the total hours of light exposure ${ }^{11,18,19}$. A study indicated that different light intensities may not exert different treatment effects on sleep problems ${ }^{20}$. In addition, a meta-analysis revealed that treatment effects did not significantly differ among patients with seasonal affective disorder when treated with strong $(6000-10,000$ lux $)$, medium (1700-3500 lux), and dim light ( $\leq 600$ lux $)^{18}$. The findings of the aforementioned studies indicate that differences in light intensity might not determine the treatment effects of light therapy. However, some recent studies have revealed that differences in light duration may exert different treatment effects on phase delay of the circadian rhythm and subjective sleepiness ${ }^{11,19}$. A longer duration of light exposure $(1-3 \mathrm{~h})$ increased the magnitude of circadian phase shift, however a higher light intensity (2000-8000 lux) did not further increase the magnitude ${ }^{11}$. Although a longer duration of light exposure $(0.2-4 \mathrm{~h})$ more effectively suppressed melatonin secretion and reduced sleepiness, the effectiveness diminished with a further increase in exposure duration ${ }^{19}$.

By examining randomized studies, previous meta-analyses have investigated the effects of pharmacological and nonpharmacological interventions on sleep-related outcomes in shift workers ${ }^{1,2}$. However, evidence regarding the effects of the dose-response and related moderators of light treatment on sleepiness and circadian phase shift in shift workers is limited. In particular, whether different intensities and durations of light exposure have different dose-response relationships with sleepiness and circadian phase shift remains unclear. The present meta-analysis examined potential variations in the dose-response effects of light therapy on sleepiness and circadian phase shift in shift workers.

\section{Methods}

This meta-analysis adhered to the criteria of the Preferred Reporting Items for Systematic Reviews and MetaAnalyses statement ${ }^{21}$.

Search strategy. Ten electronic databases, namely PubMed, CINAHL, Medline, SCOPUS, PsycInfo, Cochrane, Pubpsych, Opengrey, LILACS, and Embase, were searched for the articles for potential inclusion. The search strategy was limited to human studies and clinical trials. A specific search strategy was developed for each database on the basis of a combination of search terms (["light therapy", "phototherapy", or "light"], ["shift work", "shift", "shift worker", "work schedule tolerance", or "night work"], and ["randomized clinical trial" or "random"]). The search was conducted in June 2015, and two update searches were conducted in April 2016 and June 2020. Articles from journals and conferences were selected. In addition, no restrictions on the written language (necessary translation was performed after the search) or publication date (from inception to June 2020) were applied. The current meta-analysis used the following three-step search strategy: first, papers were searched and collected from the aforementioned databases and the relevant trial registers, references, and websites of the study organizations and institutions; second, the titles and abstracts of the searched studies were verified on the basis of inclusion criteria; and finally, data were extracted from the included studies.

Study screening. Eligibility criteria for the included studies were screened in accordance with the PICO process. Participants were individuals who were shift workers (night shift or rotating shift) or worked in simulated shifts. The interventions were those using light therapy with a single or mixed type of light. The studies made comparisons with a differentiable light intensity of $\leq 300$ lux or no active treatment in the control group (the treatment of the control group was not similar to that of the experimental group). Randomization was ensured by including experimental studies involving randomized controlled trials or randomized crossover trials. For outcomes, the included studies had adequate data for the outcome variables of sleepiness or circadian phase shift for calculating effect sizes.

Risk-of-bias assessment. Two raters independently assessed the risk of bias for each included study using the criteria provided in the Cochrane Handbook for Systematic Reviews of Interventions, Version 5.1.0 22. Each criterion was qualified as being low, high, or unclear. A study was excluded if all the criteria were identified as "high risk", which indicated that the study was not conducted in a rigorous setting. Consensus was reached through discussion.

Data extraction. Two raters independently performed the database search and screened the titles and abstracts of the retrieved studies. The two raters assessed the eligibility of the records by reviewing the full texts of the studies. Inconsistencies were resolved through discussion. The two raters independently performed data extraction and finalized the data after reaching a consensus.

Statistical analysis. The current meta-analysis used the pooled estimates (indicated by the pooled effect size) of treatment effects after combining all included studies. Extracted data were entered into Comprehensive Meta-Analysis, Version 3.0. Random-effects models were used that assumed the samples of included studies were drawn from different populations (shift workers) in which variation and estimated uncertainty were accounted for in underlying effects. The pooled effect size, indicated by Hedges's $g$ (g; $0.2-0.5=$ small effect, $0.5-0.8=$ medium effect, and $>0.8=$ large effect), was calculated using sleepiness or circadian phase shift as the outcome in shift workers. The significance level was set to $\mathrm{p}<0.05$. The directions of the outcomes were classi- 


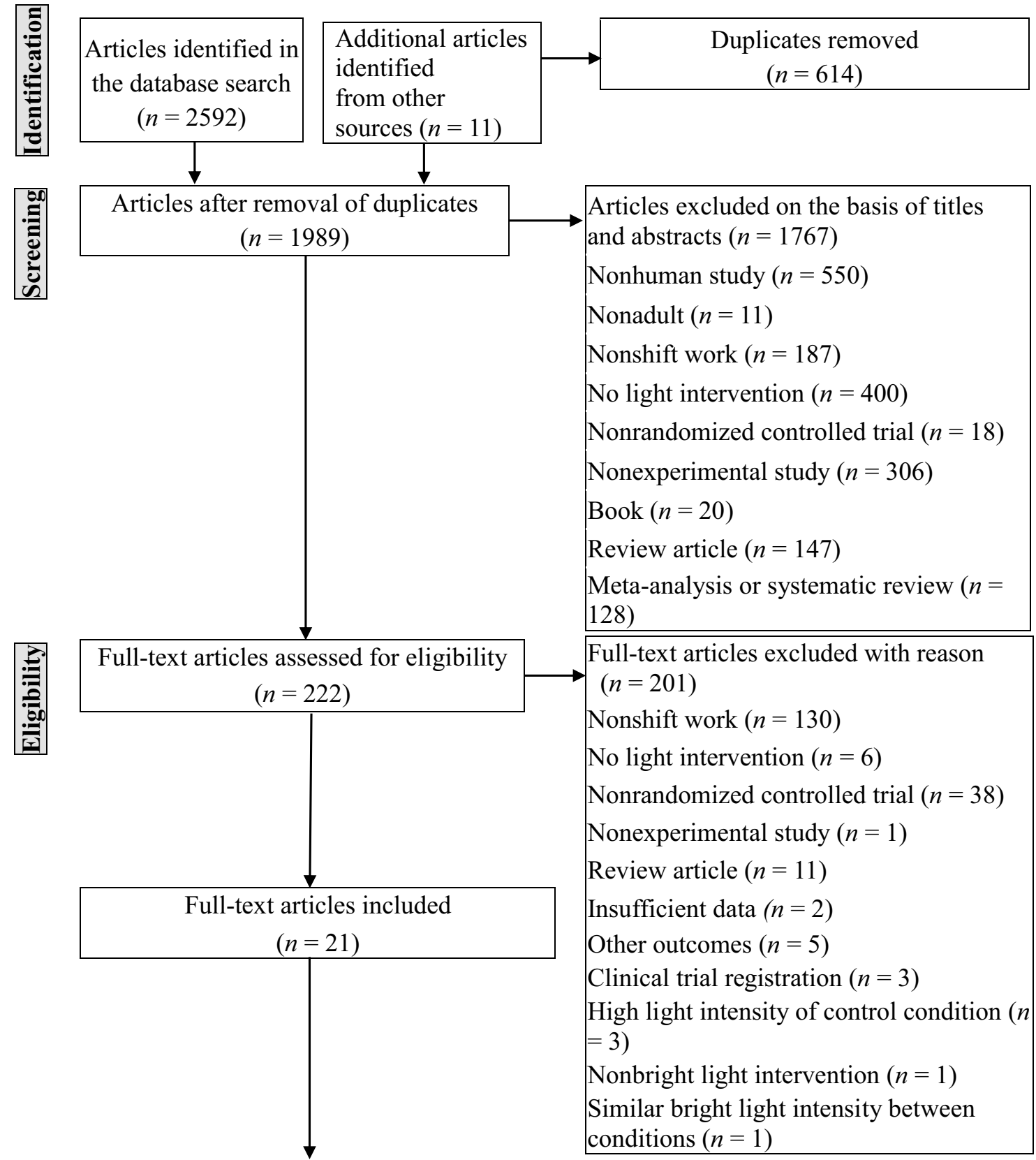

Outcomes $^{\mathrm{a}}$ :

Sleepiness $(n=15)$

Phase shift $(n=13)$

Figure 1. Flow diagram of study inclusion and exclusion in the meta-analysis a Some studies included more than one outcome.

fied as "favors light therapy" (less sleepiness or more alertness and phase delay at night or phase advance during the day) and "favors control" (no effect or opposite effects on sleepiness or phase shift). Data of each study are presented as the mean, standard deviation (SD), F test results, or $t$ test results of the experimental group and the control group for effect size calculations. The Q test and $\mathrm{I}^{2}$ statistics were used to examine betweenstudy heterogeneity, where $\mathrm{Q}>0.05$ and $\mathrm{I}^{2} \leq 50 \%$ indicated low heterogeneity and $\mathrm{I}^{2}>50 \%$ indicated substantial heterogeneity. Publication bias was examined using Egger's test. Potential publication bias was adjusted using Duval's trim-and-fill method. Sensitivity analyses were performed by removing individual studies to examine whether significance and pooled effect size were affected. Subgroup analyses with mixed-effects analysis (at least two studies were included in each subgroup) for categorical moderators and metaregression with mixed models (unrestricted maximum-likelihood) were performed to assess categorical and continuous moderators. Light 


\begin{tabular}{|c|c|c|}
\hline Characteristics & Mean & SD \\
\hline \multicolumn{3}{|l|}{ Participants $(n=656)$} \\
\hline \multirow[t]{2}{*}{ Age (years) ${ }^{\mathrm{a}}$} & 31.51 & 5.87 \\
\hline & $n$ & $\%$ \\
\hline \multicolumn{3}{|l|}{$\operatorname{Sex}^{\mathrm{a}}$} \\
\hline Female & 273 & 42.66 \\
\hline Male & 367 & 57.34 \\
\hline \multicolumn{3}{|l|}{ Sample size $^{\mathrm{b}}$} \\
\hline Experimental group & 438 & NA \\
\hline Control group & 435 & NA \\
\hline \multicolumn{3}{|l|}{ Study $(n=21)$} \\
\hline \multicolumn{3}{|l|}{ Type of shift work } \\
\hline Rotating shift & 7 & 33.33 \\
\hline Night shift & 14 & 66.67 \\
\hline \multicolumn{3}{|l|}{ Simulated shift } \\
\hline Yes & 12 & 57.14 \\
\hline No & 9 & 42.86 \\
\hline \multicolumn{3}{|l|}{ Treatment period } \\
\hline Day only or mixed time & 10 & 47.62 \\
\hline Night only & 11 & 52.38 \\
\hline \multicolumn{3}{|l|}{ Research design } \\
\hline Randomized controlled trial & 9 & 42.86 \\
\hline Randomized crossover trial & 12 & 57.14 \\
\hline \multicolumn{3}{|c|}{ Use of intention-to-treat analysis } \\
\hline Intention-to-treat & 10 & 47.62 \\
\hline Per protocol & 11 & 52.38 \\
\hline
\end{tabular}

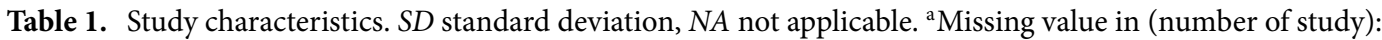
mean age (3), SD age (4), sex (1). ' Sample size: participants were included in both intervention and control groups in randomized crossover trials.

therapy dose was indicated by light intensity, duration, and their combination (i.e. lux hours $=$ lux $\times$ hours) ${ }^{23}$ for examining the dose-response effect of light therapy on sleepiness and circadian phase shift by using subgroup analyses.

Ethics declarations. No human or animal subjects were directly involved in this study.

\section{Results}

Study characteristics. A flow diagram illustrating the process of study selection (inclusion and exclusion of studies) for this meta-analysis is presented in Fig. 1. A total of 2603 articles was obtained from 10 databases and references of the articles. A total of 21 studies meeting the eligibility criteria were included ${ }^{9,13-17,24-38}$. The characteristics of the participants in the included studies are listed in Table 1 . The total number of participants was 656 , with a mean age of 31.51 years $(S D=5.87)$. The participants were shift workers or participants who performed simulated shift work during experiments. The intervention characteristics of the included studies are presented in Table 2. The studies used bright light, white light, nocturnal light, and sunlight as intervention treatments and light intensities between 430 and 10,000 lux. Furthermore, the studies used a single type of light or a combination of dim light, room light, white light, sunlight, placebo capsules, and no active treatment as the control treatment. The range of light intensity for active control treatment was 1-300 lux. The treatment duration was identical for both the experimental and control groups in each study. The total duration of exposure was between 0.5 and $42 \mathrm{~h}$. Some eligible studies included both outcomes, namely sleepiness and circadian phase shift. Fifteen studies recorded the outcome of sleepiness. These studies recorded the outcome by using the following subjective scales: the Karolinska Sleepiness Scale (KSS) with 9-point scales from "very alert" to "very sleepy, fighting sleep, an effort to stay awake;" the Stanford Sleepiness Scale (SSS) with 7-point scales from "feeling active and vital, alert, wide awake" to "lost struggle to remain awake;" and a visual analog scale (VAS) using 10-cm or $100-\mathrm{cm}$ scales with adjectives from "sleepy" to "alert". Thirteen studies recorded the outcome of circadian phase shift, which was measured by determining melatonin secretion (from a salivary or urine sample) by using a radioimmunoassay or enzyme-linked immunosorbent assay or by examining core temperature. In the current study, negative values of effect sizes represented "favors control condition," whereas positive values represented "favors light therapy." For publication bias, after Egger's test was conducted, significant publication bias was found for sleepiness $(p<0.001)$ but not for phase shift $(p=0.053)$. No potential missing study was identified for the effect of light therapy on outcomes in the funnel plot by using Duval's trim-and-fill method. 


\begin{tabular}{|c|c|c|c|c|c|c|}
\hline Study & Intervention treatment & Comparison treatment & Sample size $(n)$ & Treatment period & Shift work period & Measure period \\
\hline Babkoff 2002 & $\mathrm{BL}$ and caffeine $\mathrm{e}^{\mathrm{a}}$ & DL and caffeine & $\begin{array}{l}\text { EG: } 6 \\
\text { CG: } 6\end{array}$ & $2330-0230^{c}$ & $1730-0830^{\mathrm{fh}}$ & 0130-0430 \\
\hline Bjorvatn 2007 & $\mathrm{BL}^{\mathrm{b}}$ & Placebo capsules & $\begin{array}{l}\text { EG: } 17 \\
\text { CG: } 17\end{array}$ & $\begin{array}{l}0000-0500 \text { and } \\
1200-1430^{c}\end{array}$ & $\begin{array}{l}0630-1830 \text { and } \\
1830-0630^{\mathrm{g}}\end{array}$ & $\begin{array}{l}\text { Every } 2 \mathrm{~h} \text { from } 2000 \text { and } \\
\text { so on }\end{array}$ \\
\hline Bjorvatn 2020 & BL & DL & $\begin{array}{l}\text { EG: } 35 \\
\text { CG: } 35\end{array}$ & $\begin{array}{l}\text { 0200-0300, 0300-0400, } \\
0400-0500\end{array}$ & $2200-0600$ & every $2 \mathrm{~h}$ at $2200-0600$ \\
\hline Boivin 2012 & Intermittent $\mathrm{BL}^{\mathrm{a}}$ & No & $\begin{array}{l}\text { EG: } 8 \\
\text { CG: } 9\end{array}$ & $\begin{array}{l}6 \mathrm{~h} \text { from } 2200,2230 \\
2300 \text {, or } 2330^{c}\end{array}$ & $\begin{array}{l}8 / 8.5 \mathrm{~h} \text { between } \\
2200-0800^{\mathrm{f}}\end{array}$ & $\begin{array}{l}\text { Every } 2 \mathrm{~h} \text { (phase shift) or } \\
\text { every } 30 \mathrm{~min} \text { (sleepiness) } \\
\text { at } 0600-2200\end{array}$ \\
\hline Comtet 2019 & BL & DL & $\begin{array}{l}\text { EG: } 18 \\
\text { CG: } 18\end{array}$ & $0500-0530$ & $2000-0800$ & $0300,0500,0700,0800$ \\
\hline Dawson 1991 & $\mathrm{BL}$ and $\mathrm{DL}^{\mathrm{a}}$ & DL & $\begin{array}{l}\text { EG: } 6 \\
\text { CG: } 7\end{array}$ & $2400-0400^{c}$ & $2300-0700^{\mathrm{fh}}$ & $\begin{array}{l}0100,0300,0500 \text {, and } \\
0700\end{array}$ \\
\hline Dawson 1995 & $\mathrm{BL}^{\mathrm{a}}$ & DL & $\begin{array}{l}\text { EG: } 8 \\
\text { CG: } 8\end{array}$ & $2400-0400^{c}$ & $2300-0700^{\mathrm{fh}}$ & At night \\
\hline Griepentrog 2018 & $\mathrm{BL}$ & SL & $\begin{array}{l}\text { EG: } 26 \\
\text { CG: } 17\end{array}$ & $1900-0700$ & $1900-0700$ & 0500,0700 \\
\hline Horowitz 2001 & BL and fixed or free sleep & $\begin{array}{l}\text { RL and fixed or free } \\
\text { sleep }\end{array}$ & $\begin{array}{l}\text { EG: } 25 \\
\text { CG: } 27\end{array}$ & $2300-0700$ & $2300-0700,0700-1100$ & $2300-0700$ \\
\hline Karchani 2011 & $\mathrm{BL}^{\mathrm{b}}$ & RL & $\begin{array}{l}\text { EG: } 45 \\
\text { CG: } 45\end{array}$ & $\begin{array}{l}2200,2400,0200 \text { and } \\
0400^{c}\end{array}$ & $\begin{array}{l}0600-1400,1400-2200, \\
\text { and } 2200-0600^{\mathrm{g}} \\
\end{array}$ & $\begin{array}{l}2300,0100,0300 \text {, and } \\
0500\end{array}$ \\
\hline Kretschmer 2013 & $\mathrm{BL}$ and $\mathrm{RL}^{\mathrm{a}}$ & RL & $\begin{array}{l}\text { EG: } 16 \\
\text { CG: } 16\end{array}$ & $\begin{array}{l}2200-0200,2300-0300, \\
0000-0400^{c}\end{array}$ & $2200-0400^{\mathrm{fh}}$ & Every $2 \mathrm{~h} /$ day \\
\hline Study & Illuminance (lux) & Treatment days & Tool & Outcome & & \\
\hline Babkoff 2002 & $\begin{array}{l}\text { E: } 3000 \\
\text { C: } 20-50\end{array}$ & 1 & VAS and RIA & $\begin{array}{l}\text { Sleepiness and phase } \\
\text { shift }\end{array}$ & & \\
\hline Bjorvatn 2007 & $\begin{array}{l}\text { E: } 10,000 \\
\text { C: } 0\end{array}$ & 8 & KSS & Sleepiness & & \\
\hline Bjorvatn 2020 & $\begin{array}{l}\text { E: } 10,000 \\
\text { C: } 100\end{array}$ & 3 & KSS & Sleepiness & & \\
\hline Boivin 2012 & NA & 7 & VAS and RIA & $\begin{array}{l}\text { Sleepiness and phase } \\
\text { shift }\end{array}$ & & \\
\hline Comtet 2019 & $\begin{array}{l}\text { E: } 10,000 \\
\text { C: } 8\end{array}$ & 1 & KSS & Sleepiness & & \\
\hline Dawson 1991 & $\begin{array}{l}\text { E: BL } 6000 \text { and DL } \\
150-200 \\
\text { C: } 150-200\end{array}$ & 3 & Core temperature & Phase shift & & \\
\hline Dawson 1995 & $\begin{array}{l}\text { E: } 4000-7000 \\
\text { C: } 50\end{array}$ & 3 & RIA & Phase shift & & \\
\hline Griepentrog 2018 & $\begin{array}{l}\text { E: } 1500-2000 \\
\text { C: } 300\end{array}$ & 1 & SSS and ELISA & $\begin{array}{l}\text { Sleepiness and phase } \\
\text { shift }\end{array}$ & & \\
\hline Horowitz 2001 & $\begin{array}{l}\text { E: } 2500 \\
\text { C: } 150\end{array}$ & 3 & VAS and RIA & $\begin{array}{l}\text { Sleepiness and phase } \\
\text { shift }\end{array}$ & & \\
\hline Karchani 2011 & $\begin{array}{l}\text { E: } 2500-3000 \\
C: 300\end{array}$ & 2 & SSS & Sleepiness & & \\
\hline Kretschmer 2013 & $\begin{array}{l}\text { E: BL } 3000 \text { and RL } 300 \\
\text { C: } 300\end{array}$ & 3 & SSS & Sleepiness & & \\
\hline Study & Intervention treatment & Comparison treatment & Sample size (n) & Treatment period & Shift work period & Measure period \\
\hline Lee 2006 & BL & DL & $\begin{array}{l}\text { EG: } 11 \\
\text { CG: } 12\end{array}$ & 0045-0500 & $2300-0700$ & $\begin{array}{l}\text { Every } 30 \mathrm{~min} \text { at } \\
1530-1200 ; 0000-1800 \text { in } \\
\text { last day }\end{array}$ \\
\hline Lee 2020 & BL & DL & $\begin{array}{l}\text { EG: } 12 \\
\text { CG: } 12\end{array}$ & $0100-0600$ & $0100-0600$ & Every hour at $2100-0600$ \\
\hline Lowden 2004 & $\mathrm{BL}$ & RL & $\begin{array}{l}\text { EG: } 18 \\
\text { CG: } 18\end{array}$ & $\begin{array}{l}1515-2145,2145-0630, \\
\text { or } 2400-0630\end{array}$ & $\begin{array}{l}1515-2145,2145-0630, \\
\text { and } 2400-0630\end{array}$ & $\begin{array}{l}\text { Every } 2 \mathrm{~h} \text { at } 0000-0600 \text {, } \\
0600-1400\end{array}$ \\
\hline Nagashima 2017 & $\mathrm{BL}$ & DL & $\begin{array}{l}\text { EG: } 12 \\
\text { CG: } 12\end{array}$ & $1000-1600$ & $0000-0800$ & Every hour at $1800-0000$ \\
\hline $\begin{array}{l}\text { Sadeghniiat-Haghighi } \\
2011\end{array}$ & BL & RL & $\begin{array}{l}\text { EG: } 47 \\
\text { CG: } 47\end{array}$ & $0030-0050,0230-0250$ & $0600-1800,1800-0600^{\mathrm{g}}$ & $2200,2400,0200,0400$ \\
\hline Smith 2009 & $\begin{array}{l}\text { Intermittent BL pulse } \\
\text { and RL }\end{array}$ & RL & $\begin{array}{l}\text { EG: } 9 \\
\text { CG: } 10\end{array}$ & 0045-0400 & $2300-0700$ & Every $30 \mathrm{~min} /$ day \\
\hline Sunde 2020 & $\mathrm{BL}$ & SL & $\begin{array}{l}\text { EG: } 36 \\
\text { CG: } 36\end{array}$ & $2300-0500$ & $2300-0700$ & $\begin{array}{l}2330,0100,0230,0400, \\
0530\end{array}$ \\
\hline Tanaka 2011 & BL & No & $\begin{array}{l}\text { EG: } 61 \\
\text { CG: } 61\end{array}$ & 0730-0800 & $\begin{array}{l}0800-1700 \text {, and } \\
1630-0830\end{array}$ & 1000,1400 \\
\hline
\end{tabular}




\begin{tabular}{|c|c|c|c|c|c|c|}
\hline Study & Intervention treatment & Comparison treatment & Sample size (n) & Treatment period & Shift work period & Measure period \\
\hline Thorne 2010 & $\begin{array}{l}\text { WL and wearing sun- } \\
\text { glasses }\end{array}$ & No & $\begin{array}{l}\text { EG: } 10 \\
\text { CG: } 10\end{array}$ & $1300-1400$ & $\begin{array}{l}1800-0600, \text { or } \\
1900-0700\end{array}$ & Every $4 \mathrm{~h} /$ day \\
\hline Yoon 2002 & BL & RL & $\begin{array}{l}\text { EG: } 12 \\
\text { CG: } 12\end{array}$ & $\begin{array}{l}0100-0500 \text { and } \\
0830-0930\end{array}$ & $2200-0800$ & 4 times at $2400-0600$ \\
\hline Study & Illuminance (lux) & Treatment days & Tool & Outcome & & \\
\hline Lee 2006 & $\begin{array}{l}\text { E: } 3500 \\
\text { C: }<50\end{array}$ & 2 & RIA & Phase shift & & \\
\hline Lee 2020 & $\begin{array}{l}\text { E: } 430 \\
C:<1\end{array}$ & 1 & KSS and RIA & $\begin{array}{l}\text { Sleepiness and phase } \\
\text { shift }\end{array}$ & & \\
\hline Lowden 2004 & $\begin{array}{l}\text { E: } 2500 \\
\text { C: } 300\end{array}$ & 15 & KSS and RIA & $\begin{array}{l}\text { Sleepiness and phase } \\
\text { shift }\end{array}$ & & \\
\hline Nagashima 2017 & $\begin{array}{l}\text { E: }>3000 \\
C:<50\end{array}$ & 1 & RIA & Phase shift & & \\
\hline $\begin{array}{l}\text { Sadeghniiat-Haghighi } \\
2011\end{array}$ & $\begin{array}{l}\text { E: } 2500 \\
\text { C: } 300\end{array}$ & 1 & SSS & Sleepiness & & \\
\hline Smith 2009 & $\begin{array}{l}\text { E: } 4100 \\
\text { C: }<50\end{array}$ & 8 & RIA & Phase shift & & \\
\hline Sunde 2020 & $\begin{array}{l}\text { E: } 900 \\
\text { C: } 90\end{array}$ & 3 & KSS and ELISA & $\begin{array}{l}\text { Sleepiness and phase } \\
\text { shift }\end{array}$ & & \\
\hline Tanaka 2011 & $\begin{array}{l}\text { E: } 5444-8826 \\
\text { C: } 0\end{array}$ & 30 & KSS & Sleepiness & & \\
\hline Thorne 2010 & $\begin{array}{l}\text { E: } 3000 \\
\text { C: } 0\end{array}$ & 4 & RIA & Phase shift & & \\
\hline Yoon 2002 & $\begin{array}{l}\text { E: NL } 4000-6000 \text { and SL } \\
10,000 \\
C: R L<200 \text { and } S L \\
10,000\end{array}$ & 3 & VAS & Sleepiness & & \\
\hline
\end{tabular}

Table 2. Intervention characteristics of the included studies. $B L$ bright light, $D L$ dim light, $W L$ white light, $N L$ nocturnal light, $S L$ sunlight, $R L$ room light, $E$ experimental group, $C$ control group, No no treatment, NA not available, ELISA enzyme-linked immunosorbent assay, KSS Karolinska Sleepiness Scale, RIA Radioimmunoassay, SSS Standford Sleepiness Scale, VAS Visual Analog Scale.

Risk-of-bias assessment. The risk-of-bias assessment of the included studies is shown in Table 3. None of the studies met all the criteria. Most of the studies (all $k=19)$ had an unclear or high risk in terms of sequence generation, allocation concealment, blinding of participants and personnel, and blinding of outcome assessors. Subgroup analysis was not performed for these criteria because a substantial risk of bias was already demonstrated in these assessments. Five studies had a high risk of incomplete outcome data, and one study had a high risk of selective outcome reporting. The remaining studies had a low level of risk for the two criteria. Thus, subgroup analysis was performed for only incomplete outcome data for both outcomes: sleepiness (Table 5c), low risk $(\mathrm{g}=0.474,95 \%$ confidence interval $[\mathrm{CI}]=0.089-0.290, \mathrm{p}<0.001)$ and high risk $(\mathrm{g}=0.190,95 \% \mathrm{CI}=$ $0.339-0.609, \mathrm{p}<0.001)$; phase shift (Table 6c), low risk $(\mathrm{g}=1.150,95 \% \mathrm{CI}=0.718-1.583, \mathrm{p}<0.001)$ and high risk $(g=0.857,95 \% \mathrm{CI}=0.223-1.491, \mathrm{p}=0.008)$. Overall, these results indicate that the risk of selective outcome reporting was low; however, the other five criteria of bias substantially influenced the effects of the outcomes.

Treatment effects. The results of random-effects models used for examining the effects of light therapy on sleepiness and circadian phase shift in shift workers are presented in Figs. 2 and 3, respectively. For sleepiness, light therapy had a small to medium pooled effect size $(g=0.429,95 \%$ CI $0.290-0.569, p<0.001)$ in the 15 included studies. The between-study heterogeneity was low $\left(\mathrm{Q}=20.784, \mathrm{p}=0.107, \mathrm{I}^{2}=32.641\right)$. The effects of light therapy on reducing sleepiness were significant in nine studies but not in other six studies. Sensitivity analysis showed that the pooled effect size remained significant after each study was removed $(\mathrm{g}=0.381-0.481$, all $\mathrm{p}<0.001)$. For phase shift, the pooled effect size was large $(g=1.079,95 \%$ CI $0.723-1.434, p<0.001)$ in the 13 included studies. The between-study heterogeneity was substantial $\left(Q=27.372, p=0.007, I^{2}=56.159\right)$. Significantly positive effects of light therapy were observed in 10 studies but not in the other 3 studies. Similarly, sensitivity analysis showed that the pooled effect size remained similar and significant after each study was removed ( $g=0.980$ to 1.161 , all $\mathrm{p}<0.001)$.

Moderator analyses. The moderators are listed in Table 4. The results of subgroup and metaregression analyses are presented in Tables $5 \mathrm{a}-\mathrm{c}, 6 \mathrm{a}-\mathrm{c}$ and 7, respectively. Overall, the subgroup analysis performed using mixed-effects models demonstrated low heterogeneity $\left(\mathrm{I}^{2}=0-30.014\right)$. The metaregression analysis performed using mixed models exhibited low heterogeneity for the models of sleepiness $\left(\mathrm{I}^{2}=0-36.55\right)$ and the significant moderators of phase shift $\left(\mathrm{I}^{2}=29.04-39.68\right)$ but substantial heterogeneity for the nonsignificant moderators of phase shift $\left(\mathrm{I}^{2}=59.67-62.44\right)$.

Moderator analyses of dose-response effects. The dose-response effect of light therapy on sleepiness was significantly associated with different subgroups of light intensity, treatment duration, and number of daily treatment sessions (Table 5a). Higher to lower effect sizes were associated with medium, high, and 


\begin{tabular}{|c|c|c|c|c|c|c|}
\hline Study & $\begin{array}{l}\text { Random sequence } \\
\text { generation }\end{array}$ & Allocation concealment & $\begin{array}{l}\text { Blinding of participants } \\
\text { and personnel }\end{array}$ & $\begin{array}{l}\text { Blinding of outcome } \\
\text { assessment }\end{array}$ & $\begin{array}{l}\text { Incomplete outcome } \\
\text { data }\end{array}$ & Selective reporting \\
\hline Babkoff 2002 & $\mathrm{U}$ & $\mathrm{U}$ & $\mathrm{H}$ & $\mathrm{L}$ & $\mathrm{H}$ & $\mathrm{L}$ \\
\hline Bjorvatn 2007 & $\mathrm{U}$ & $\mathrm{U}$ & $\mathrm{U}$ & $\mathrm{U}$ & $\mathrm{H}$ & $\mathrm{L}$ \\
\hline Bjorvatn 2020 & $\mathrm{U}$ & $\mathrm{L}$ & $\mathrm{L}$ & $\mathrm{U}$ & $\mathrm{H}$ & $\mathrm{L}$ \\
\hline Boivin 2012 & $\mathrm{U}$ & $\mathrm{U}$ & $\mathrm{U}$ & $\mathrm{U}$ & $\mathrm{L}$ & $\mathrm{H}$ \\
\hline Comtet 2019 & $\mathrm{~L}$ & $\mathrm{U}$ & $\mathrm{U}$ & $\mathrm{U}$ & $\mathrm{L}$ & $\mathrm{L}$ \\
\hline Dawson 1991 & $\mathrm{U}$ & $\mathrm{U}$ & $\mathrm{U}$ & $\mathrm{U}$ & $\mathrm{L}$ & $\mathrm{L}$ \\
\hline Dawson 1995 & $\mathrm{U}$ & $\mathrm{U}$ & $\mathrm{L}$ & $\mathrm{U}$ & $\mathrm{L}$ & $\mathrm{L}$ \\
\hline Griepentrog 2018 & $\mathrm{H}$ & $\mathrm{U}$ & $\mathrm{U}$ & $\mathrm{U}$ & $\mathrm{L}$ & $\mathrm{L}$ \\
\hline Horowitz 2001 & $\mathrm{U}$ & $\mathrm{U}$ & $\mathrm{U}$ & $\mathrm{U}$ & $\mathrm{L}$ & $\mathrm{L}$ \\
\hline Karchani 2011 & $\mathrm{U}$ & $\mathrm{U}$ & $\mathrm{U}$ & $\mathrm{U}$ & $\mathrm{L}$ & $\mathrm{L}$ \\
\hline Kretschmer 2013 & $\mathrm{U}$ & $\mathrm{U}$ & $\mathrm{U}$ & $\mathrm{U}$ & $\mathrm{L}$ & $\mathrm{L}$ \\
\hline Lee 2006 & $\mathrm{U}$ & $\mathrm{U}$ & $\mathrm{U}$ & $\mathrm{U}$ & $\mathrm{L}$ & $\mathrm{L}$ \\
\hline Lee 2020 & $\mathrm{U}$ & $\mathrm{U}$ & $\mathrm{U}$ & $\mathrm{U}$ & $\mathrm{L}$ & $\mathrm{L}$ \\
\hline Lowden 2004 & $\mathrm{U}$ & $\mathrm{U}$ & $\mathrm{H}$ & $\mathrm{U}$ & $\mathrm{L}$ & $\mathrm{L}$ \\
\hline Nagashima 2017 & $\mathrm{U}$ & $\mathrm{U}$ & $\mathrm{U}$ & $\mathrm{U}$ & $\mathrm{L}$ & $\mathrm{L}$ \\
\hline $\begin{array}{l}\text { Sadeghniiat-Haghighi } \\
2011\end{array}$ & $\mathrm{U}$ & $\mathrm{U}$ & $\mathrm{U}$ & $\mathrm{U}$ & $\mathrm{L}$ & $\mathrm{L}$ \\
\hline Smith 2009 & $\mathrm{U}$ & $\mathrm{U}$ & $\mathrm{U}$ & $\mathrm{L}$ & $\mathrm{L}$ & $\mathrm{L}$ \\
\hline Sunde 2020 & $\mathrm{U}$ & $\mathrm{U}$ & $\mathrm{U}$ & $\mathrm{U}$ & $\mathrm{H}$ & $\mathrm{L}$ \\
\hline Tanaka 2011 & $\mathrm{~L}$ & $\mathrm{~L}$ & $\mathrm{H}$ & $\mathrm{U}$ & $\mathrm{L}$ & $\mathrm{L}$ \\
\hline Thorne 2010 & $\mathrm{U}$ & $\mathrm{U}$ & $\mathrm{H}$ & $\mathrm{U}$ & $\mathrm{H}$ & $\mathrm{L}$ \\
\hline Yoon 2002 & $\mathrm{U}$ & $\mathrm{U}$ & $\mathrm{U}$ & $\mathrm{U}$ & $\mathrm{L}$ & $\mathrm{L}$ \\
\hline
\end{tabular}

Table 3. Risk-of-Bias assessment of included studies. $L$ low risk, $H$ high risk, $U$ unclear risk.

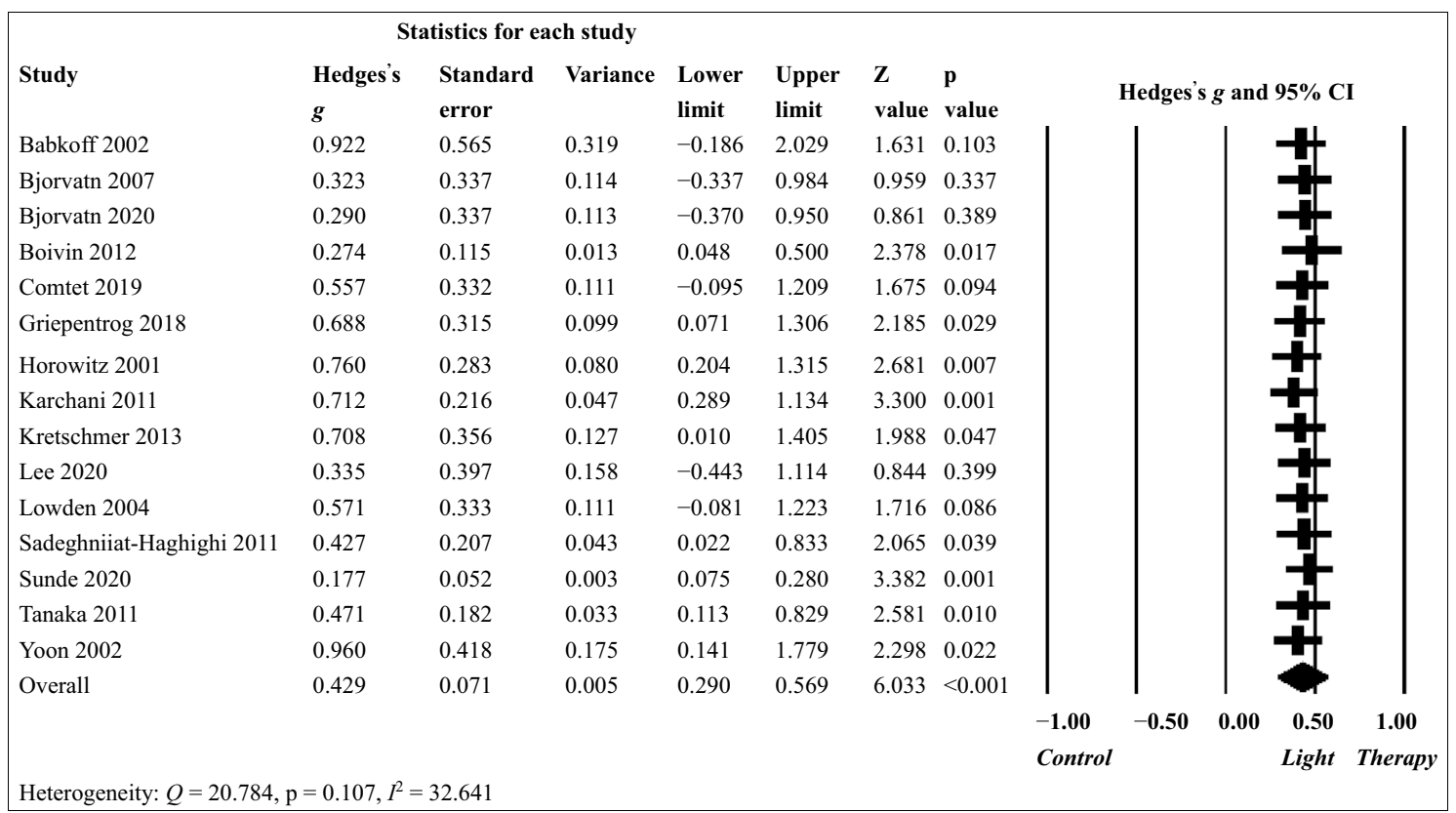

Figure 2. Treatment effect of light therapy on sleepiness in shift workers analyzed using a random-effects model.

low light intensity when light intensity was grouped as follows: (1) 1000-5000 lux ( $\mathrm{g}=0.632,95 \% \mathrm{CI}=0.423-$ $0.842, \mathrm{p}<0.001),>5000 \operatorname{lux}(\mathrm{g}=0.482,95 \% \mathrm{CI}=0.234-0.730, \mathrm{p}<0.001)$, and $<1000 \operatorname{lux}(\mathrm{g}=0.180,95 \% \mathrm{CI}=$ $0.078-0.282, \mathrm{p}<0.001)$; (2) 2000-5000 lux ( $\mathrm{g}=0.625,95 \% \mathrm{CI}=0.402-0.848, \mathrm{p}<0.001)$, $>5000 \operatorname{lux}(\mathrm{g}=0.482$, $95 \% \mathrm{CI}=0.234-0.730, \mathrm{p}<0.001)$, and $<2000 \mathrm{lux}(\mathrm{g}=0.194,95 \% \mathrm{CI}=0.093-0.294, \mathrm{p}<0.001)$; and (3) 3000$5000 \operatorname{lux}(\mathrm{g}=0.764,95 \% \mathrm{CI}=0.359-1.168, \mathrm{p}<0.001),>5000 \operatorname{lux}(\mathrm{g}=0.482,95 \% \mathrm{CI}=0.234-0.730, \mathrm{p}<0.001)$, and $<3000$ lux $(g=0.240,95 \% \mathrm{CI}=0.146-0.334, \mathrm{p}<0.001)$. A higher effect size was associated with a shorter treatment duration as follows: $(1)$ daily treatment duration $\leq 1 \mathrm{~h}(\mathrm{~g}=0.504,95 \% \mathrm{CI}=0.312-0.695, \mathrm{p}<0.001)$, and daily treatment duration $>1 \mathrm{~h}(\mathrm{~g}=0.400,95 \% \mathrm{CI}=0.191-0.608, \mathrm{p}<0.001)$ and $(2)$ treatment duration in 


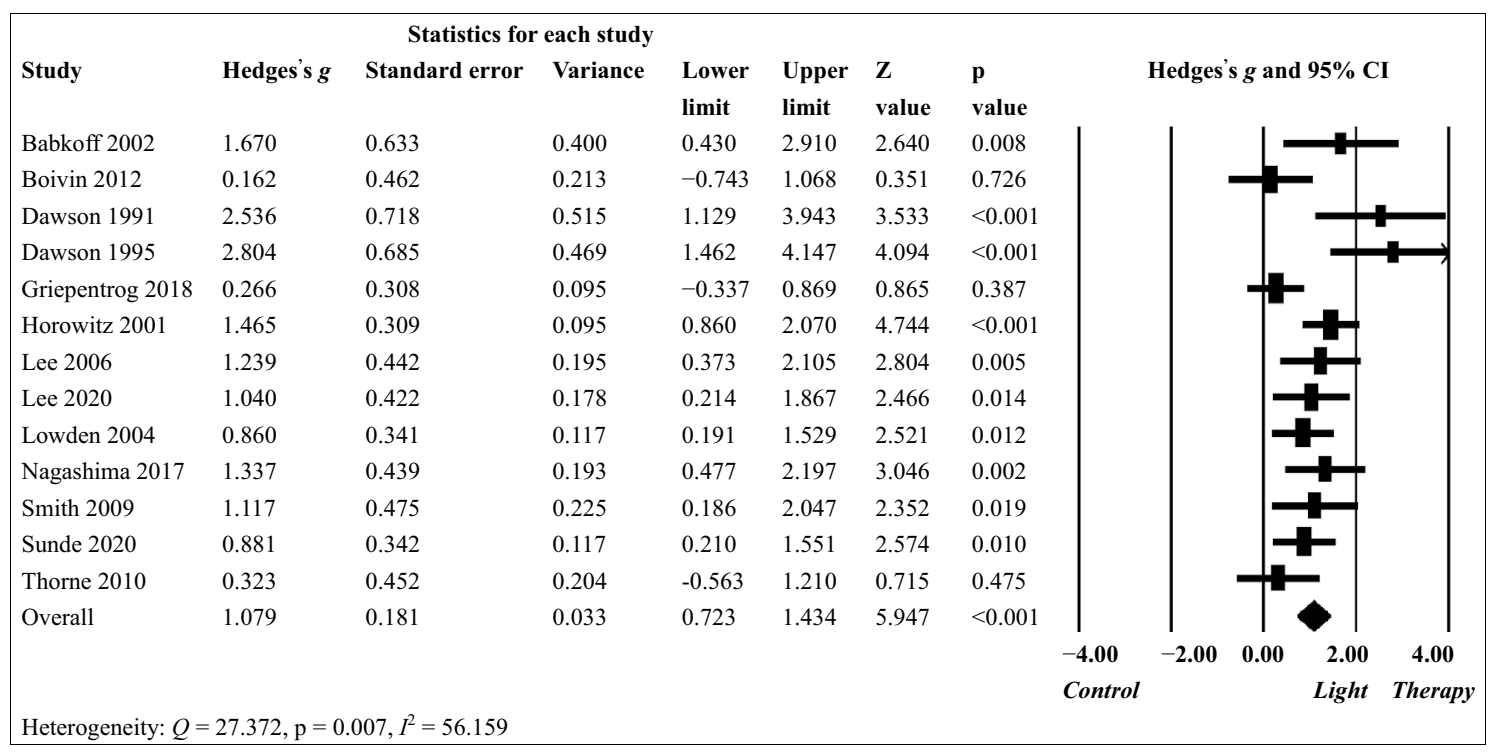

Figure 3. Treatment effect of light therapy on phase shift in shift workers analyzed using a random-effects model.

total $\leq 10 \mathrm{~h}(\mathrm{~g}=0.532,95 \% \mathrm{CI}=0.361-0.703, \mathrm{p}<0.001)$ and treatment duration in total $>10 \mathrm{~h}(\mathrm{~g}=0.309,95 \% \mathrm{CI}$ $=0.095-0.523, \mathrm{p}=0.005)$. A relatively higher effect size was associated with a higher number of daily treatment session: session $>1(\mathrm{~g}=0.535,95 \% \mathrm{CI}=0.261-0.809, \mathrm{p}<0.001)$ and session $=1(\mathrm{~g}=0.400,95 \% \mathrm{CI}=0.240-0.560$, $\mathrm{p}<0.001$ ). However, continuous moderators of lux hours, light intensity, treatment duration, mean age, percentage of female participants, and sample size on sleepiness were not found to be significant in metaregression analyses (all $p>0.05$, Table 7). These results suggest nonlinear effects and potential saturation of light intensity (medium lux) and treatment duration (lower duration) for achieving a more favorable effect of light therapy on sleepiness, whereas a higher number of treatment sessions was more effective.

The dose-response effect of light therapy on phase shift was significantly associated with different subgroups of light intensity, treatment duration, and number of daily treatment sessions (Table 6a). Higher to lower effect sizes were associated with high, medium, and low light intensity when light intensity was grouped as follows: (1) $>5000 \operatorname{lux}(\mathrm{g}=2.676,95 \% \mathrm{CI}=1.705-3.648, \mathrm{p}<0.001), 1000-5000 \operatorname{lux}(\mathrm{g}=0.983,95 \% \mathrm{CI}=0.611-1.356$, $\mathrm{p}<0.001)$, and $<1000 \operatorname{lux}(\mathrm{g}=0.944,95 \% \mathrm{CI}=0.423-1.465, \mathrm{p}<0.001) ;(2)>5000 \operatorname{lux}(\mathrm{g}=2.676,95 \% \mathrm{CI}=$ $1.705-3.648, \mathrm{p}<0.001), 2000-5000 \operatorname{lux}(\mathrm{g}=1.134,95 \% \mathrm{CI}=0.829-1.440, \mathrm{p}<0.001)$, and $<2000 \operatorname{lux}(\mathrm{g}=0.677$, $95 \% \mathrm{CI}=0.199-1.154, \mathrm{p}=0.005)$; and $(3)>5000 \operatorname{lux}(\mathrm{g}=2.676,95 \% \mathrm{CI}=1.705-3.648, \mathrm{p}<0.001), 3000-5000$ lux $(\mathrm{g}=1.204,95 \% \mathrm{CI}=0.853-1.555, \mathrm{p}<0.001)$, and $<3000 \operatorname{lux}(\mathrm{g}=0.710,95 \% \mathrm{CI}=0.362-1.058, \mathrm{p}<0.001)$. A higher effect size was associated with a longer daily treatment duration: $>1 \mathrm{~h}(\mathrm{~g}=1.166,95 \% \mathrm{CI}=0.688-1.644, \mathrm{p}<0.001)$ and $\leq 1 \mathrm{~h}(\mathrm{~g}=0.952,95 \% \mathrm{CI}=0.206-1.698, \mathrm{p}=0.012)$. However, a slightly higher effect size was associated with a shorter total treatment duration: $\leq 10 \mathrm{~h}(\mathrm{~g}=1.182,95 \% \mathrm{CI}=0.865-1.499, \mathrm{p}<0.001)$, and $>10 \mathrm{~h}(\mathrm{~g}=1.166,95 \%$ $\mathrm{CI}=0.282-2.050, \mathrm{p}=0.010$ ). A slightly higher effect size was associated with a fewer number of daily treatment session: session $=1(\mathrm{~g}=1.137,95 \% \mathrm{CI}=0.607-1.667, \mathrm{p}<0.001)$ and session $>1(\mathrm{~g}=1.130,95 \% \mathrm{CI}=0.627-1.633$, $\mathrm{p}<0.001)$. Significant effects of continuous moderators on phase shift were observed in metaregression analyses (Table 7): lux hours (coefficient $=0.014,95 \% \mathrm{CI}=0.004-0.024, \mathrm{p}=0.008)$, light intensity (coefficient $=0.286,95 \%$ $\mathrm{CI}=0.061-0.512, \mathrm{p}=0.013$ ), and mean age (coefficient $=-0.052,95 \% \mathrm{CI}=-0.092$ to $-0.013, \mathrm{p}=0.009$ ); however, the effects were not significant for the percentage of female participants and sample size (all $p>0.05)$. These results suggest a positive association between higher light intensity and better light therapy effect on phase shift, and fewer treatment sessions were slightly more beneficial. However, the effect of treatment duration on phase shift was inconclusive. The effect of lux hours was lower than that of light intensity. These results indicat that the treatment duration did not exert a positive effect on phase shift. Moreover, younger participants experienced better treatment effects on phase shift than did older participants.

Moderator analyses of treatment settings and research design. For sleepiness, the treatment effect was significant in the following subgroups (Table 5b): (1) timing of treatment, nighttime $(\mathrm{g}=0.427,95 \%$ $\mathrm{CI}=0.170-0.683, \mathrm{p}=0.001)$ and daytime or mixed $(\mathrm{g}=0.431,95 \% \mathrm{CI}=0.279-0.583, \mathrm{p}<0.001) ;(2)$ treatment day, same day $(\mathrm{g}=0.575,95 \% \mathrm{CI}=0.347-0.804, \mathrm{p}<0.001)$ and different day $(\mathrm{g}=0.363,95 \% \mathrm{CI}=0.203-0.522$, $\mathrm{p}<0.001$ ); (3) type of treatment light, single type ( $\mathrm{g}=0.361,95 \% \mathrm{CI}=0.228-0.494, \mathrm{p}<0.001)$ and mixed type $(\mathrm{g}=0.779,95 \% \mathrm{CI}=0.401-1.158, \mathrm{p}<0.001)$; (4) control treatment, active treatment $(\mathrm{g}=0.511,95 \% \mathrm{CI}=$ $0.312-0.710, \mathrm{p}<0.001)$ and no treatment $(\mathrm{g}=0.330,95 \% \mathrm{CI}=0.401-1.158, \mathrm{p}<0.001)$; (5) shift work, night shift $(\mathrm{g}=0.439,95 \% \mathrm{CI}=0.195-0.684, \mathrm{p}<0.001)$ and rotating shift $(\mathrm{g}=0.430,95 \% \mathrm{CI}=0.284-0.576, \mathrm{p}<0.001) ;(6)$ simulated shift, simulated $(\mathrm{g}=0.454,95 \% \mathrm{CI}=0.162-0.746, \mathrm{p}=0.002)$ and nonsimulated $(\mathrm{g}=0.431,95 \% \mathrm{CI}=$ $0.290-0.573, \mathrm{p}<0.001)$; (7) timing of measure, nighttime $(\mathrm{g}=0.463,95 \% \mathrm{CI}=0.225-0.702, \mathrm{p}<0.001)$ and day time or mixed $(\mathrm{g}=0.410,95 \% \mathrm{CI}=0.252-0.569, \mathrm{p}<0.001)$; $(8)$ research design, randomized controlled trial 


\begin{tabular}{|c|c|c|c|c|c|c|c|c|c|c|}
\hline Study & $\begin{array}{l}\text { Age (mean or } \\
\text { range) }\end{array}$ & Female (n) & $\begin{array}{l}\text { Sample size } \\
\text { (n) }\end{array}$ & Shift work & $\begin{array}{l}\text { Simulated } \\
\text { shift }\end{array}$ & $\begin{array}{l}\text { Research } \\
\text { design }\end{array}$ & $\begin{array}{l}\text { Data } \\
\text { handling }\end{array}$ & $\begin{array}{l}\text { Timing of } \\
\text { treatment }\end{array}$ & $\begin{array}{l}\text { Treatment } \\
\text { day }\end{array}$ & \begin{tabular}{|l|}
$\begin{array}{l}\text { Measure } \\
\text { period }\end{array}$ \\
\end{tabular} \\
\hline Babkoff 2002 & 24.6 & 5 & 12 & Night & Simulated & RCT & $\begin{array}{l}\text { PP or } \\
\text { unknown }\end{array}$ & Night time & Same day & Night time \\
\hline Bjorvatn 2007 & 42 & 1 & 17 & Rotating & No & Crossover & $\begin{array}{l}\text { PP or } \\
\text { unknown }\end{array}$ & Day or mixed & Different day & Night time \\
\hline Bjorvatn 2020 & 35.4 & 28 & 35 & Night & No & Crossover & $\begin{array}{l}\text { PP or } \\
\text { unknown }\end{array}$ & Night time & Different day & Day or mixed \\
\hline Boivin 2012 & 30.06 & 8 & 17 & Rotating & No & RCT & ITT & Day or mixed & Different day & Day or mixed \\
\hline Comtet 2019 & 24.78 & 5 & 18 & Night & Simulated & Crossover & $\begin{array}{l}\begin{array}{l}\text { PP or } \\
\text { unknown }\end{array} \\
\end{array}$ & Day or mixed & Same day & Day or mixed \\
\hline Dawson 1991 & 21.2 & 6 & 13 & Night & Simulated & RCT & ITT & Night time & Different day & Night time \\
\hline Dawson 1995 & 23.6 & NA & 16 & Night & Simulated & RCT & ITT & Night time & Different day & Night time \\
\hline Griepentrog 2018 & $26-32$ & 22 & 43 & Night & No & Crossover & ITT & Day or mixed & Same day & Night time \\
\hline Horowitz 2001 & 26.99 & 27 & 54 & Rotating & Simulated & RCT & $\begin{array}{l}\text { PP or } \\
\text { unknown }\end{array}$ & Day or mixed & Different day & Day or mixed \\
\hline Karchani 2011 & 30.415 & 0 & 90 & Rotating & No & Crossover & ITT & Night time & Same day & Night time \\
\hline Kretschmer 2013 & 58.5 & 50 & 32 & Night & Simulated & RCT & ITT & Night time & Different day & Day or mixed \\
\hline Lee 2006 & 24.5 & 12 & 23 & Night & Simulated & RCT & $\begin{array}{l}\text { PP or } \\
\text { unknown }\end{array}$ & Night time & Different day & Day or mixed \\
\hline Lee 2020 & 20.65 & 0 & 24 & Night & Simulated & Crossover & $\begin{array}{l}\text { PP or } \\
\text { unknown }\end{array}$ & Day or mixed & Same day & Night time \\
\hline Lowden 2004 & 36.2 & 1 & 18 & Rotating & No & Crossover & ITT & Day or mixed & Different day & Day or mixed \\
\hline Nagashima 2017 & 24.8 & 0 & 12 & Night & Simulated & Crossover & \begin{tabular}{|l|}
$\begin{array}{l}\text { PP or } \\
\text { unknown }\end{array}$ \\
\end{tabular} & Day or mixed & Same day & Night time \\
\hline $\begin{array}{l}\text { Sadeghniiat- } \\
\text { Haghighi } 2011\end{array}$ & 33 & 0 & 94 & Rotating & No & Crossover & $\begin{array}{l}\text { PP or } \\
\text { unknown }\end{array}$ & Night time & Same day & Night time \\
\hline Smith 2009 & 25.79 & 11 & 19 & Night & Simulated & RCT & ITT & Night time & Different day & Day or mixed \\
\hline Sunde 2020 & $19-30$ & 24 & 36 & Night & Simulated & Crossover & $\begin{array}{l}\text { PP or } \\
\text { unknown }\end{array}$ & Night time & Different day & Night time \\
\hline Tanaka 2011 & 29.7 & 61 & 61 & Rotating & No & Crossover & ITT & Day or mixed & Different day & Day or mixed \\
\hline Thorne 2010 & 46.5 & 0 & 10 & Night & No & Crossover & $\begin{array}{l}\text { PP or } \\
\text { unknown }\end{array}$ & Day or mixed & Different day & Night time \\
\hline Yoon 2002 & $21-24$ & 12 & 12 & Night & No & Crossover & ITT & Day or mixed & Different day & Night time \\
\hline Study & $\begin{array}{l}\text { Control } \\
\text { intervention }\end{array}$ & $\begin{array}{l}\text { Treatment } \\
\text { light }\end{array}$ & \begin{tabular}{|l} 
Light \\
intensity of \\
EG (lux)
\end{tabular} & $\begin{array}{l}\text { Treatment } \\
\text { duration } \\
\text { (daily hour) }\end{array}$ & $\begin{array}{l}\text { Treatment } \\
\text { duration } \\
\text { (total hour) }\end{array}$ & $\begin{array}{l}\text { Daily } \\
\text { treatment } \\
\text { session (n) }\end{array}$ & $\begin{array}{l}\text { Lux-hours } \\
\text { (per 1000) }\end{array}$ & & & \\
\hline Babkoff 2002 & Dim light & Mixed & 3000 & 1 & 1 & 1 & 3.00 & & & \\
\hline Bjorvatn 2007 & No & Single & 10,000 & 0.5 & 4 & 1 & 40.00 & & & \\
\hline Bjorvatn 2020 & Dim Red light & Single & 10,000 & 0.5 & 1.5 & 1 & 15.00 & & & \\
\hline Boivin 2012 & No & Single & NA & 6 & 42 & 1 & NA & & & \\
\hline Comtet 2019 & Dim light & Single & 10,000 & 0.5 & 0.5 & 1 & 5.00 & & & \\
\hline Dawson 1991 & Dim light & Single & 6175 & 8 & 24 & 1 & 148.20 & & & \\
\hline Dawson 1995 & Dim light & Single & 5500 & 4 & 12 & 1 & 66.00 & & & \\
\hline Griepentrog 2018 & Standard light & Mixed & 1750 & 10 & 10 & 1 & 17.50 & & & \\
\hline Horowitz 2001 & Room light & Single & 2500 & 6 & 18 & 1 & 45.00 & & & \\
\hline Karchani 2011 & Room light & Single & 2750 & 1 & 1 & 4 & 2.75 & & & \\
\hline Kretschmer 2013 & Room light & Mixed & 3300 & 2 & 6 & 1 & 19.80 & & & \\
\hline Lee 2006 & Dim light & Single & 3500 & 1.25 & 2.5 & 5 & 8.75 & & & \\
\hline Lee 2020 & Dim light & Single & 430 & 4.17 & 4.17 & 5 & 1.79 & & & \\
\hline Lowden 2004 & Room light & Single & 2500 & NA & NA & NA & NA & & & \\
\hline Nagashima 2017 & Dim light & Single & 3000 & 6 & 6 & 1 & 18.00 & & & \\
\hline $\begin{array}{l}\text { Sadeghniiat- } \\
\text { Haghighi } 2011\end{array}$ & Room light & Single & 2500 & 0.67 & 0.67 & 2 & 1.68 & & & \\
\hline Smith 2009 & Room light & Mixed & 4100 & 1 & 8 & 4 & 32.80 & & & \\
\hline Sunde 2020 & Standard light & Single & 900 & 6 & 18 & 1 & 16.20 & & & \\
\hline Tanaka 2011 & No & Single & 7135 & 0.17 & 5 & 1 & 35.68 & & & \\
\hline Thorne 2010 & No & Single & 3000 & 1 & 4 & 1 & 12.00 & & & \\
\hline Yoon 2002 & Room light & Mixed & 15,000 & 5 & 15 & 1 & 225.00 & & & \\
\hline
\end{tabular}

Table 4. Moderators used in subgroup and metaregression analyses. RCT randomized controlled trial, Crossover randomized crossover trial, ITT intention-to-treat, $P P$ per protocol. 


\begin{tabular}{|c|c|c|c|c|c|c|}
\hline \multicolumn{7}{|l|}{ (a) } \\
\hline \multirow[b]{2}{*}{ Subgroup } & \multirow[b]{2}{*}{ k } & \multirow[b]{2}{*}{ Hedges's g } & \multicolumn{2}{|l|}{ 95\% CI } & \multirow[b]{2}{*}{$\mathrm{p}(\mathrm{Z})^{\mathrm{a}}$} & \multirow[b]{2}{*}{$\mathrm{I}^{2}$} \\
\hline & & & Lower & Upper & & \\
\hline \multicolumn{7}{|c|}{ Intensity of light treatment (lux) } \\
\hline \multicolumn{7}{|c|}{ Group 1} \\
\hline$<1000$ & 2 & 0.180 & 0.078 & 0.282 & $<0.001$ & 0 \\
\hline $1000-5000$ & 7 & 0.632 & 0.423 & 0.842 & $<0.001$ & 0 \\
\hline$>5000$ & 5 & 0.482 & 0.234 & 0.730 & $<0.001$ & 0 \\
\hline \multicolumn{7}{|l|}{ Group 2} \\
\hline$<2000$ & 3 & 0.194 & 0.093 & 0.294 & $<0.001$ & 0 \\
\hline $2000-5000$ & 6 & 0.625 & 0.402 & 0.848 & $<0.001$ & 0 \\
\hline$>5000$ & 5 & 0.482 & 0.234 & 0.730 & $<0.001$ & 0 \\
\hline \multicolumn{7}{|l|}{ Group 3} \\
\hline$<3000$ & 6 & 0.240 & 0.146 & 0.334 & $<0.001$ & 0 \\
\hline $3000-5000$ & 3 & 0.764 & 0.359 & 1.168 & $<0.001$ & 0 \\
\hline$>5000$ & 5 & 0.482 & 0.234 & 0.730 & $<0.001$ & 0 \\
\hline \multicolumn{7}{|l|}{ Treatment duration (daily hr) } \\
\hline$\leq 1$ & 7 & 0.504 & 0.312 & 0.695 & $<0.001$ & 0 \\
\hline$>1$ & 7 & 0.400 & 0.191 & 0.608 & $<0.001$ & 0 \\
\hline \multicolumn{7}{|l|}{ Treatment duration (total hr) } \\
\hline$\leq 10$ & 10 & 0.532 & 0.361 & 0.703 & $<0.001$ & 0 \\
\hline$>10$ & 4 & 0.309 & 0.095 & 0.523 & 0.005 & 27.071 \\
\hline \multicolumn{7}{|c|}{ Number of daily treatment sessions (n) } \\
\hline 1 & 11 & 0.400 & 0.240 & 0.560 & $<0.001$ & 0 \\
\hline$>1$ & 3 & 0.535 & 0.261 & 0.809 & $<0.001$ & 0 \\
\hline \multicolumn{7}{|l|}{ (b) } \\
\hline \multirow[b]{2}{*}{ Subgroup } & \multirow[b]{2}{*}{$\mathbf{k}$} & \multirow[b]{2}{*}{ Hedges's g } & \multicolumn{2}{|l|}{$95 \% \mathrm{CI}$} & & \\
\hline & & & Lower & Upper & $\mathbf{p}(\mathrm{Z})^{\mathrm{a}}$ & $\mathrm{I}^{2}$ \\
\hline Timing of treatment & & & & & & \\
\hline Night time & 6 & 0.427 & 0.170 & 0.683 & 0.001 & 0 \\
\hline Day time or mixed & 9 & 0.431 & 0.279 & 0.583 & $<0.001$ & 0 \\
\hline Treatment day & & & & & & \\
\hline Same day & 6 & 0.575 & 0.347 & 0.804 & $<0.001$ & 0 \\
\hline Different day & 9 & 0.363 & 0.203 & 0.522 & $<0.001$ & 0 \\
\hline Type of treatment light & & & & & & \\
\hline Single type of light & 11 & 0.361 & 0.228 & 0.494 & $<0.001$ & 0 \\
\hline Mixed type of light & 4 & 0.779 & 0.401 & 1.158 & $<0.001$ & 0 \\
\hline Control treatment & & & & & & \\
\hline Active treatment & 12 & 0.511 & 0.312 & 0.710 & $<0.001$ & 0 \\
\hline No treatment & 3 & 0.330 & 0.146 & 0.513 & $<0.001$ & 0 \\
\hline Shift work & & & & & & \\
\hline Night shift & 8 & 0.439 & 0.195 & 0.684 & $<0.001$ & 0 \\
\hline Rotating shift & 7 & 0.430 & 0.284 & 0.576 & $<0.001$ & 0 \\
\hline Simulated shift & & & & & & \\
\hline Yes & 6 & 0.454 & 0.162 & 0.746 & 0.002 & 0 \\
\hline No & 9 & 0.431 & 0.290 & 0.573 & $<0.001$ & 0 \\
\hline Timing of measure & & & & & & \\
\hline Night time & 8 & 0.463 & 0.225 & 0.702 & $<0.001$ & 0 \\
\hline Day time or mixed & 7 & 0.410 & 0.252 & 0.569 & $<0.001$ & 0 \\
\hline Research design & & & & & & \\
\hline Randomized controlled trial & 4 & 0.502 & 0.185 & 0.820 & 0.002 & 0 \\
\hline Randomized crossover trial & 11 & 0.426 & 0.257 & 0.595 & $<0.001$ & 0 \\
\hline Data handling & & & & & & \\
\hline Continued & & & & & & \\
\hline
\end{tabular}




\begin{tabular}{|c|c|c|c|c|c|c|}
\hline \multicolumn{7}{|l|}{ (b) } \\
\hline \multirow[b]{2}{*}{ Subgroup } & \multirow[b]{2}{*}{$\mathbf{k}$} & \multirow[b]{2}{*}{ Hedges's $\mathrm{g}$} & \multicolumn{2}{|c|}{ 95\% CI } & \multirow[b]{2}{*}{$\mathbf{p}(\mathbf{Z})^{\mathbf{a}}$} & \multirow[b]{2}{*}{$I^{2}$} \\
\hline & & & Lower & Upper & & \\
\hline Intention-to-treat & 7 & 0.480 & 0.310 & 0.649 & $<0.001$ & 0 \\
\hline Per protocol or unknown & 8 & 0.305 & 0.149 & 0.461 & $<0.001$ & 0 \\
\hline \multicolumn{7}{|l|}{ (c) } \\
\hline \multirow[b]{2}{*}{ Subgroup } & \multirow[b]{2}{*}{ k } & \multirow[b]{2}{*}{ Hedges's g } & \multicolumn{2}{|l|}{ 95\% CI } & \multirow[b]{2}{*}{$\mathbf{p}(\mathrm{Z})^{\mathrm{a}}$} & \multirow[b]{2}{*}{$\mathrm{I}^{2}$} \\
\hline & & & Lower & Upper & & \\
\hline \multicolumn{7}{|l|}{ Subjective scale } \\
\hline SSS & 4 & 0.602 & 0.354 & 0.849 & $<0.001$ & 0 \\
\hline VAS & 4 & 0.576 & 0.190 & 0.963 & 0.003 & 0 \\
\hline KSS & 7 & 0.221 & 0.127 & 0.315 & $<0.001$ & 0 \\
\hline \multicolumn{7}{|l|}{ Risk of bias } \\
\hline \multicolumn{7}{|l|}{ Incomplete outcome data } \\
\hline Low risk & 11 & 0.474 & 0.339 & 0.609 & $<0.001$ & 0 \\
\hline High risk & 4 & 0.190 & 0.089 & 0.290 & $<0.001$ & 0 \\
\hline
\end{tabular}

Table 5. Moderators of light treatment effect on sleepiness by using subgroup analyses with mixed-effects models. ${ }^{\text {a }}$ value for the $\mathrm{Z}$ test of the moderator effect.

$(\mathrm{g}=0.502,95 \% \mathrm{CI}=0.185-0.820, \mathrm{p}=0.002)$ and randomized crossover trial $(\mathrm{g}=0.426,95 \% \mathrm{CI}=0.257-0.595$, $\mathrm{p}<0.001)$; and (9) data handling, intention-to-treat $(\mathrm{g}=0.480,95 \% \mathrm{CI}=0.310-0.649, \mathrm{p}<0.001)$ and per protocol or unknown ( $g=0.305,95 \% \mathrm{CI}=0.149-0.461, \mathrm{p}<0.001)$.

For phase shift, the treatment effect was significant in most of the following subgroups (Table 6b): (1) timing of treatment, nighttime $(\mathrm{g}=1.528,95 \% \mathrm{CI}=0.950-2.107, \mathrm{p}<0.001)$ and daytime or mixed $(\mathrm{g}=0.798,95 \%$ $\mathrm{CI}=0.390-1.206, \mathrm{p}<0.001) ;(2)$ treatment day, same day $(\mathrm{g}=0.967,95 \% \mathrm{CI}=0.334-1.599, \mathrm{p}=0.003)$, different day $(\mathrm{g}=1.137,95 \% \mathrm{CI}=0.688-1.586, \mathrm{p}<0.001)$; (3) type of treatment light, single type $(\mathrm{g}=1.141,95 \% \mathrm{CI}=$ $0.741-1.541, \mathrm{p}<0.001)$ and mixed type $(\mathrm{g}=0.889,95 \% \mathrm{CI}=0.062-1.717, \mathrm{p}=0.035) ;(4)$ control treatment, active treatment $(\mathrm{g}=1.216,95 \% \mathrm{CI}=0.848-1.584, \mathrm{p}<0.001)$, but not significant for no treatment $(\mathrm{g}=0.245,95 \% \mathrm{CI}$ $=-0.389-0.878, \mathrm{p}=0.449) ;(5)$ shift work, night shift $(\mathrm{g}=1.164,95 \% \mathrm{CI}=0.720-1.607, \mathrm{p}<0.001)$ and rotating shift $(g=0.892,95 \%$ CI $=0.193-1.592, p=0.012)$; (6) simulated shift, simulated $(g=1.379,95 \% \mathrm{CI}=1.041-1.718$, $\mathrm{p}<0.001)$ and nonsimulated $(\mathrm{g}=0.436,95 \% \mathrm{CI}=0.071-0.802, \mathrm{p}=0.019)$; (7) timing of measure, nighttime $(\mathrm{g}=1.194,95 \% \mathrm{CI}=0.635-1.753, \mathrm{p}<0.001)$ and daytime or mixed $(\mathrm{g}=1.016,95 \% \mathrm{CI}=0.592-1.441, \mathrm{p}<0.001)$; (8) research design, randomized controlled trial $(\mathrm{g}=1.447,95 \% \mathrm{CI}=0.869-2.025, \mathrm{p}<0.001)$, randomized crossover trial $(\mathrm{g}=0.748,95 \% \mathrm{CI}=0.423-1.072, \mathrm{p}<0.001)$; (9) data handling, intention-to-treat $(\mathrm{g}=1.128,95 \% \mathrm{CI}=$ $0.398-1.859, \mathrm{p}=0.002)$ and per protocol or unknown $(\mathrm{g}=1.127,95 \% \mathrm{CI}=0.827-1.429, \mathrm{p}<0.001)$. These results suggest that treatment settings and research design significantly influenced treatment effects.

Moderator analyses of subjective scales. For the subjective scales of sleepiness, higher to lower effect sizes were associated with SSS, VAS, and KSS, respectively (Table 5c): SSS ( $g=0.602,95 \%$ CI $=0.354-0.849$, $\mathrm{p}<0.001)$, VAS $(\mathrm{g}=0.576,95 \% \mathrm{CI}=0.190-0.963, \mathrm{p}=0.003)$, and $\mathrm{KSS}(\mathrm{g}=0.221,95 \% \mathrm{CI}=0.127-0.315, \mathrm{p}<0.001)$. The findings suggest potential inconsistency in the use of different scales in assessing sleepiness.

\section{Discussion}

The current meta-analysis is the first to investigate the dose-response relationship between light therapy and the circadian phase in shift workers. The results of random-effects models revealed that the pooled effect size of light therapy in reducing sleepiness (15 studies) was small to medium ( $g=0.429)$ and phase shift (13 studies) was large $(\mathrm{g}=1.079)$ in shift workers. In addition, we observed a significant dose-response effect of light therapy on both outcomes, where different intensities and durations of light therapy exerted different effects on sleepiness and phase shift. In particular, light therapy that involved a medium light intensity, shorter duration, and more daily treatment sessions was significantly more effective in reducing sleepiness. These findings suggest a potential saturation of medium light intensity and shorter treatment duration in reducing sleepiness. By contrast, higherintensity light therapy was significantly more effective in phase shifting; however, whether a longer or shorter treatment duration exerted a better effect on phase shifting remained unclear.

A study on light therapy in healthy participants reported no significant differences in the effects of moderate and high light intensities and longer duration on circadian phase shift ${ }^{11}$. Another study reported that a longer duration of light exposure more effectively reduced sleepiness than shorter durations did $^{19}$. The findings of the current meta-analysis reveal different effects of light therapy on sleepiness and phase shift in shift workers. A moderate intensity and shorter duration of light were more effective in reducing sleepiness, whereas a higher intensity but an unknown duration of light were more effective in sleep phase shifting. The inconsistencies in the results of the current and previous studies may be due to the differences in study populations (inclusion of healthy participants without shift work in previous studies versus those of simulated shift and shift workers in the current study). Furthermore, a higher number of treatment sessions was more effective in reducing sleepiness, 


\begin{tabular}{|c|c|c|c|c|c|c|}
\hline \multicolumn{7}{|l|}{ (a) } \\
\hline \multirow[b]{2}{*}{ Subgroup } & \multirow[b]{2}{*}{$\mathbf{k}$} & \multirow[b]{2}{*}{ Hedges's g } & \multicolumn{2}{|l|}{ 95\% CI } & \multirow[b]{2}{*}{$\mathbf{p}(\mathbf{Z})^{\mathrm{a}}$} & \multirow[b]{2}{*}{$\mathbf{I}^{2}$} \\
\hline & & & Lower & Upper & & \\
\hline \multicolumn{7}{|c|}{ Intensity of light treatment (lux) } \\
\hline \multicolumn{7}{|c|}{ Group 1} \\
\hline$<1000$ & 2 & 0.944 & 0.423 & 1.465 & $<0.001$ & 0 \\
\hline $1000-5000$ & 8 & 0.983 & 0.611 & 1.356 & $<0.001$ & 0 \\
\hline$>5000$ & 2 & 2.676 & 1.705 & 3.648 & $<0.001$ & 0 \\
\hline \multicolumn{7}{|l|}{ Group 2} \\
\hline$<2000$ & 3 & 0.677 & 0.199 & 1.154 & 0.005 & 0 \\
\hline $2000-5000$ & 7 & 1.134 & 0.829 & 1.440 & $<0.001$ & 0 \\
\hline$>5000$ & 2 & 2.676 & 1.705 & 3.648 & $<0.001$ & 0 \\
\hline \multicolumn{7}{|l|}{ Group 3} \\
\hline$<3000$ & 4 & 0.710 & 0.362 & 1.058 & $<0.001$ & 0 \\
\hline $3000-5000$ & 6 & 1.204 & 0.853 & 1.555 & $<0.001$ & 0 \\
\hline$>5000$ & 2 & 2.676 & 1.705 & 3.648 & $<0.001$ & 0 \\
\hline \multicolumn{7}{|l|}{ Treatment duration (daily hr) } \\
\hline$\leq 1$ & 3 & 0.952 & 0.206 & 1.698 & 0.012 & 0.743 \\
\hline$>1$ & 9 & 1.166 & 0.688 & 1.644 & $<0.001$ & 17.873 \\
\hline \multicolumn{7}{|l|}{ Treatment duration (total hr) } \\
\hline$\leq 10$ & 7 & 1.182 & 0.865 & 1.499 & $<0.001$ & 0 \\
\hline$>10$ & 5 & 1.166 & 0.282 & 2.050 & 0.010 & 30.014 \\
\hline \multicolumn{7}{|c|}{ Number of daily treatment sessions (n) } \\
\hline 1 & 9 & 1.137 & 0.607 & 1.667 & $<0.001$ & 18.492 \\
\hline$>1$ & 3 & 1.130 & 0.627 & 1.633 & $<0.001$ & 0 \\
\hline \multicolumn{7}{|l|}{ (b) } \\
\hline \multirow[b]{2}{*}{ Subgroup } & \multirow[b]{2}{*}{$\mathbf{k}$} & \multirow[b]{2}{*}{ Hedges's g } & \multicolumn{2}{|l|}{ 95\% CI } & & \\
\hline & & & Lower & Upper & $\mathbf{p}(\mathrm{Z})^{\mathbf{a}}$ & $\mathbf{I}^{2}$ \\
\hline Timing of treatment & & & & & & \\
\hline Night time & 6 & 1.528 & 0.950 & 2.107 & $<0.001$ & 7.083 \\
\hline Day time or mixed & 7 & 0.798 & 0.390 & 1.206 & $<0.001$ & 0 \\
\hline Treatment day & & & & & & \\
\hline Same day & 4 & 0.967 & 0.334 & 1.599 & 0.003 & 0 \\
\hline Different day & 9 & 1.137 & 0.688 & 1.586 & $<0.001$ & 22.871 \\
\hline Type of treatment light & & & & & & \\
\hline Single type of light & 10 & 1.141 & 0.741 & 1.541 & $<0.001$ & 21.004 \\
\hline Mixed type of light & 3 & 0.889 & 0.062 & 1.717 & 0.035 & 0 \\
\hline Control treatment & & & & & & \\
\hline Active treatment & 11 & 1.216 & 0.848 & 1.584 & $<0.001$ & 8.3649 \\
\hline No treatment & 2 & 0.245 & -0.389 & 0.878 & 0.449 & 0 \\
\hline Shift work & & & & & & \\
\hline Night shift & 10 & 1.164 & 0.720 & 1.607 & $<0.001$ & 11.668 \\
\hline Rotating shift & 3 & 0.892 & 0.193 & 1.592 & 0.012 & 5.7388 \\
\hline Simulated shift & & & & & & \\
\hline Yes & 9 & 1.379 & 1.041 & 1.718 & $<0.001$ & 7.0895 \\
\hline No & 4 & 0.436 & 0.071 & 0.802 & 0.019 & 0 \\
\hline Timing of measure & & & & & & \\
\hline Night time & 8 & 1.194 & 0.635 & 1.753 & $<0.001$ & 16.256 \\
\hline Day time or mixed & 5 & 1.016 & 0.592 & 1.441 & $<0.001$ & 0.110 \\
\hline Research design & & & & & & \\
\hline Randomized controlled trial & 7 & 1.447 & 0.869 & 2.025 & $<0.001$ & 14.171 \\
\hline Randomized crossover trial & 6 & 0.748 & 0.423 & 1.072 & $<0.001$ & 0 \\
\hline Data handling & & & & & & \\
\hline Continued & & & & & & \\
\hline
\end{tabular}




\begin{tabular}{|c|c|c|c|c|c|c|}
\hline \multicolumn{7}{|l|}{ (b) } \\
\hline \multirow[b]{2}{*}{ Subgroup } & \multirow[b]{2}{*}{$\mathbf{k}$} & \multirow[b]{2}{*}{ Hedges's g } & \multicolumn{2}{|l|}{ 95\% CI } & \multirow[b]{2}{*}{$\mathbf{p}(\mathbf{Z})^{\mathbf{a}}$} & \multirow[b]{2}{*}{$I^{2}$} \\
\hline & & & Lower & Upper & & \\
\hline Intention-to-treat & 6 & 1.128 & 0.398 & 1.859 & 0.002 & 26.446 \\
\hline Per protocol or unknown & 7 & 1.127 & 0.827 & 1.429 & $<0.001$ & 0 \\
\hline \multicolumn{7}{|l|}{ (c) } \\
\hline \multirow[b]{2}{*}{ Subgroup } & \multirow[b]{2}{*}{$\mathbf{k}$} & \multirow[b]{2}{*}{ Hedges's g } & \multicolumn{2}{|l|}{ 95\% CI } & \multirow[b]{2}{*}{$\mathbf{p}(\mathrm{Z})^{\mathrm{a}}$} & \multirow[b]{2}{*}{$I^{2}$} \\
\hline & & & Lower & Upper & & \\
\hline \multicolumn{7}{|l|}{ Risk of bias } \\
\hline \multicolumn{7}{|l|}{ Incomplete outcome data } \\
\hline Low risk & 10 & 1.150 & 0.718 & 1.583 & $<0.001$ & 15.024 \\
\hline High risk & 3 & 0.857 & 0.223 & 1.491 & 0.008 & 9.249 \\
\hline
\end{tabular}

Table 6. Moderators of light treatment effect on phase shift by using subgroup analyses with mixed-effects models. ${ }^{\text {a }} \mathrm{P}$ value for the $\mathrm{Z}$ test of the moderator effect.

whereas a lower number of treatment sessions was more favorable for sleep phase shifting. The current findings are based on the pooled effect sizes of eligible studies on light therapy; thus, they may serve as a reference for establishing the effectiveness of light therapy and its dose-response relationship with sleepiness and circadian phase in shift workers.

Shift workers are usually more prone to sleepiness than nonshift workers are ${ }^{39}$. Light therapy may suppress melatonin secretion and reduce sleepiness in shift workers ${ }^{11,12}$. Moreover, light therapy may shift the circadian phase for sleeping later on workdays and sleeping earlier on days off. In addition, the effects of light therapy on sleepiness varied with the type of shift work. Light therapy was significantly more effective at reducing sleepiness and inducing phase shifting in the night shift than in the rotating shift; this difference may be due to steady changes in the circadian phase experienced by night shift workers. Furthermore, light therapy was more effective at reducing sleepiness and inducing phase shifting in simulated shift workers possibly due to changes initiated in the circadian phase by simulated shifts and because they were more adapted to shift work than nonsimulated shift participants were. Furthermore, light therapy was more effective at reducing sleepiness and inducing phase shifting in simulated shift workers possibly due to changes initiated in the circadian phase by simulated shifts and because they were more adapted to shift work than nonsimulated shift participants were. Therefore, light therapy was more effective at restoring alertness to normal levels and inducing phase shifting in simulated shift workers. Circadian phase shift was characterized by a change in the circadian phase from irregular to relatively normal during the night shift. Moreover, quality assessments indicated that substantially high or unclear risks of methodological biases affected both outcomes in the included studies. Particularly, pooled effect sizes were lower for studies with a high risk of incomplete outcome data. In addition, light therapy exerted better effects on sleepiness and phase shifting in studies that included a randomized controlled design, active control treatment, timing of measure at night, a mixed type of light (for sleepiness) or a single type of light (for phase shifting), treatment at night (for phase shifting) or other times (for sleepiness), and treatment on the same day (for sleepiness) or different day (for phase shifting). Younger people may benefit more in phase shifting after receiving light therapy. These results may serve as a reference for the design and settings of future studies on light therapy.

This meta-analysis had some limitations, and its results must be interpreted with caution. The included studies had high interstudy variation regarding the degree of intensity, duration of treatment, and number of treatment sessions of light therapy; this variation did not provide a sufficient layer of treatment dose of light therapy for a meta-analysis. Currently, we can only report dose-response patterns of a higher or lower degree of treatment doses of light therapy that may be more effective on sleepiness and phase shift in shift workers instead of making conclusive recommendations for the most effective treatment dose. In addition, we examined the effects of different treatment settings and research design on outcomes. These findings may serve as a reference for future experimental studies on the effective treatment dose of light therapy. Regarding data quality, we performed a subgroup analysis to reveal the reasons for potential heterogeneity in pooled effect sizes. Low heterogeneity was observed for most of the significant subgroups of the included studies. Most of the included studies had small sample sizes, and slight variations in studies could change the results. Potential publication bias was found in studies on sleepiness. Therefore, we used random-effects models for adjusting interstudy variations. During data collection, two studies were excluded because of insufficient data, a problem that persisted even after the authors were contacted. Many identified studies were not randomized; therefore, the number of eligible studies in this meta-analysis was limited. In this meta-analysis, we focused on sleepiness and circadian phase shift; however, their intersection could not be examined. For example, the effect of light therapy on changes in sleepiness may be influenced by underlying phase resetting. Moreover, the effect sizes of sleepiness may be influenced by different subjective scales used in the included studies. We performed a subgroup analysis examining this problem, which suggested significant differences among the scales. The outcomes of sleep disorders in shift workers were not investigated. Meta-analyses and experimental studies have insufficiently examined whether light therapy is effective in treating sleep disorders in shift workers. 


\begin{tabular}{|c|c|c|c|c|c|c|}
\hline \multirow[b]{2}{*}{ Moderator } & \multirow[b]{2}{*}{$\mathbf{k}$} & \multirow[b]{2}{*}{$\beta$} & \multicolumn{2}{|l|}{$95 \% \mathrm{CI}$} & \multirow[b]{2}{*}{$\mathbf{p}(\mathrm{Z})^{\mathrm{a}}$} & \multirow[b]{2}{*}{$\mathbf{I}^{2}$} \\
\hline & & & Lower & Upper & & \\
\hline \multicolumn{7}{|l|}{ Outcome-sleepiness } \\
\hline Lux-hours (per 1000) & 13 & 0.002 & -0.002 & 0.006 & 0.282 & 36.55 \\
\hline Light intensity (lux per 1000) & 13 & 0.018 & -0.025 & 0.061 & 0.404 & 18.55 \\
\hline Treatment duration (daily hr) & 14 & -0.019 & -0.069 & 0.031 & 0.453 & 18.81 \\
\hline Treatment duration (total hr) & 14 & -0.007 & -0.017 & 0.004 & 0.192 & 29.12 \\
\hline Age (mean) & 12 & 0.003 & -0.019 & 0.024 & 0.805 & 0 \\
\hline Female (\%) & 15 & -0.003 & -0.389 & 0.382 & 0.986 & 33.83 \\
\hline Sample size (n) & 15 & 0.002 & -0.003 & 0.008 & 0.457 & 28.86 \\
\hline \multicolumn{7}{|l|}{ Outcome-phase shift } \\
\hline Lux-hours (per 1000) & 11 & 0.014 & 0.004 & 0.024 & 0.008 & 35.51 \\
\hline Light intensity (lux per 1000) & 11 & 0.286 & 0.061 & 0.512 & 0.013 & 39.68 \\
\hline Treatment duration (daily hr) & 12 & -0.033 & -0.173 & 0.108 & 0.649 & $60.54^{\star}$ \\
\hline Treatment duration (total hr) & 12 & -0.010 & -0.047 & \begin{tabular}{|l|}
0.027 \\
\end{tabular} & 0.596 & $62.44^{*}$ \\
\hline Age (mean) & 11 & -0.052 & -0.092 & -0.013 & 0.009 & 29.04 \\
\hline Female (\%) & 12 & 0.154 & -1.162 & 1.470 & 0.819 & $50.48^{\star}$ \\
\hline Sample size (n) & 13 & -0.007 & -0.034 & 0.021 & 0.627 & $59.67^{*}$ \\
\hline
\end{tabular}

Table 7. Continuous moderators of light treatment effect on sleepiness and phase shift by using metaregression with mixed models. ${ }^{*}$ Significant at $\mathrm{p}<0.05$. ${ }^{\mathrm{P}} \mathrm{P}$ value for the $\mathrm{Z}$ test of the moderator effect.

\section{Conclusion}

The findings of the current meta-analysis suggest that exposure to a moderate intensity and a shorter duration of light therapy was relatively better at reducing sleepiness in shift workers (such as shift nurses) compared with lower- and higher-intensity and longer-duration light therapy, respectively. This finding suggests potential saturation of the treatment dose of light therapy on sleepiness. Exposure to higher-intensity light was more effective at inducing phase shifting compared with low- and moderate-intensity light, but the most effective treatment duration was unclear. Shift workers may mitigate sleepiness and shift sleep phases to achieve better sleep by exposing themselves to bright light at night to improve alertness at work and shift their circadian phase for improved sleep.

Received: 12 March 2020; Accepted: 13 April 2021

Published online: 07 June 2021

\section{References}

1. Liira, J. et al. Pharmacological interventions for sleepiness and sleep disturbances caused by shift work. Cochrane Database Syst. Rev. 8, CD009776. https://doi.org/10.1002/14651858.CD009776.pub2 (2014).

2. Slanger, T. E. et al. Person-directed, non-pharmacological interventions for sleepiness at work and sleep disturbances caused by shift work. Cochrane Database Syst. Rev. https://doi.org/10.1002/14651858.CD010641.pub2 (2016).

3. International Labour Organization. C171-Night Work Convention, 1990 (No. 171). http://www.ilo.org/dyn/normlex/en/f?p= NORMLEXPUB:12100:0::NO::P12100_INSTRUMENT_ID:312316 (1990).

4. Chang, Y. S. et al. Rotating night shifts too quickly may cause anxiety and decreased attentional performance, and impact prolactin levels during the subsequent day: A case control study. BMC Psychiatry 14, 218. https://doi.org/10.1186/s12888-014-0218-7 (2014).

5. Rajaratnam, S. M., Howard, M. E. \& Grunstein, R. R. Sleep loss and circadian disruption in shift work: Health burden and management. Med. J. Aust. 199, S11-S15 (2013).

6. Richter, K., Acker, J., Adam, S. \& Niklewski, G. Prevention of fatigue and insomnia in shift workers-A review of non-pharmacological measures. EPMA J. 7, 16. https://doi.org/10.1186/s13167-016-0064-4 (2016).

7. Vedaa, O. et al. Prospective study of predictors and consequences of insomnia: Personality, lifestyle, mental health, and work-related stressors. Sleep Med. 20, 51-58. https://doi.org/10.1016/j.sleep.2015.12.002 (2016).

8. Shahid, A., Shen, J. \& Shapiro, C. M. Measurements of sleepiness and fatigue. J. Psychosom. Res. 69, 81-89 (2010).

9. Kretschmer, V., Schmidt, K. H. \& Griefahn, B. Bright-light effects on cognitive performance in elderly persons working simulated night shifts: Psychological well-being as a mediator?. Int. Arch. Occup. Environ. Health 86, 901-914. https://doi.org/10.1007/ s00420-012-0826-9 (2013).

10. Garbarino, S. et al. Sleepiness and sleep disorders in shift workers: A study on a group of Italian police officers. Sleep 25, 648-653 (2002).

11. Dewan, K., Benloucif, S., Reid, K., Wolfe, L. F. \& Zee, P. C. Light-induced changes of the circadian clock of humans: Increasing duration is more effective than increasing light intensity. Sleep 34, 593-599 (2011).

12. Sack, R. L. et al. Circadian rhythm sleep disorders: Part I, basic principles, shift work and jet lag disorders. An American Academy of Sleep Medicine review. Sleep 30, 1460-1483 (2007).

13. Smith, M. R., Fogg, L. F. \& Eastman, C. I. Practical interventions to promote circadian adaptation to permanent night shift work: Study 4. J. Biol. Rhythms 24, 161-172. https://doi.org/10.1177/0748730409332068 (2009).

14. Dawson, D. \& Campbell, S. S. Timed exposure to bright light improves sleep and alertness during simulated night shifts. Sleep 14, $511-516(1991)$.

15. Dawson, D., Encel, N. \& Lushington, K. Improving adaptation to simulated night shift: Timed exposure to bright light versus daytime melatonin administration. Sleep 18, 11-21 (1995).

16. Babkoff, H., French, J., Whitmore, J. \& Sutherlin, R. Single-dose bright light and/or caffeine effect on nocturnal performance. Aviat. Space Environ. Med. 73, 341-350 (2002). 
17. Lowden, A., Akerstedt, T. \& Wibom, R. Suppression of sleepiness and melatonin by bright light exposure during breaks in night work. J. Sleep Res. 13, 37-43 (2004).

18. Lee, T. M. \& Chan, C. C. Dose-response relationship of phototherapy for seasonal affective disorder: A meta-analysis. Acta Psychiatr. Scand. 99, 315-323 (1999).

19. Chang, A. M. et al. Human responses to bright light of different durations. J. Physiol. 590, 3103-3112. https://doi.org/10.1113/ jphysiol.2011.226555 (2012).

20. Ando, K., Kripke, D. F., Cole, R. J. \& Elliott, J. A. Light mask 500 lux treatment for delayed sleep phase syndrome. Prog. Neuropsychopharmacol. Biol. Psychiatry 23, 15-24 (1999).

21. Moher, D., Liberati, A., Tetzlaff, J. \& Altman, D. G. Preferred reporting items for systematic reviews and meta-analyses: The PRISMA statement. Ann. Intern. Med. 151, 264-269. https://doi.org/10.7326/0003-4819-151-4-200908180-00135 (2009).

22. Higgins, J. P. T. \& Green, S. Cochrane Handbook for Systematic Reviews of Interventions (The Cochrane Collaboration, 2008).

23. Golden, R. N. et al. The efficacy of light therapy in the treatment of mood disorders: A review and meta-analysis of the evidence. Am. J. Psychiatry 162, 656-662 (2005).

24. Bjorvatn, B., Pallesen, S., Waage, S., Thun, E. \& Blytt, K. M. The effects of bright light treatment on subjective and objective sleepiness during three consecutive night shifts among hospital nurses-A counter-balanced placebo-controlled crossover study. Scand. J. Work Environ. Health https://doi.org/10.5271/sjweh.3930 (2020).

25. Bjorvatn, B. et al. Randomized placebo-controlled field study of the effects of bright light and melatonin in adaptation to night work. Scand. J. Work Environ. Health 33, 204-214 (2007).

26. Boivin, D. B., Boudreau, P. \& Tremblay, G. M. Phototherapy and orange-tinted goggles for night-shift adaptation of police officers on patrol. Chronobiol. Int. 29, 629-640. https://doi.org/10.3109/07420528.2012.675252 (2012).

27. Comtet, H. et al. Light therapy with boxes or glasses to counteract effects of acute sleep deprivation. Sci. Rep. 9, 18073. https://doi. org/10.1038/s41598-019-54311-x (2019).

28. Griepentrog, J. E., Labiner, H. E., Gunn, S. R. \& Rosengart, M. R. Bright environmental light improves the sleepiness of nightshift ICU nurses. Crit. Care 22, 295. https://doi.org/10.1186/s13054-018-2233-4 (2018).

29. Horowitz, T. S., Cade, B. E., Wolfe, J. M. \& Czeisler, C. A. Efficacy of bright light and sleep/darkness scheduling in alleviating circadian maladaptation to night work. Am. J. Physiol. Endocrinol. Metab. 281, E384-E391 (2001).

30. Karchani, M., Kakooei, H., Yazdi, Z. \& Zare, M. Do bright-light shock exposures during breaks reduce subjective sleepiness in night workers?. Sleep Biol. Rhythms 9, 95-102. https://doi.org/10.1111/j.1479-8425.2011.00490.x (2011).

31. Lee, C., Smith, M. R. \& Eastman, C. I. A compromise phase position for permanent night shift workers: Circadian phase after two night shifts with scheduled sleep and light/dark exposure. Chronobiol. Int. 23, 859-875. https://doi.org/10.1080/074205206008271 60 (2006).

32. Lee, S. I. et al. Melatonin suppression during a simulated night shift in medium intensity light is increased by 10 -minute breaks in dim light and decreased by 10-minute breaks in bright light. Chronobiol. Int. https://doi.org/10.1080/07420528.2020.1752704 (2020).

33. Nagashima, S. et al. Bright-light exposure during daytime sleeping affects nocturnal melatonin secretion after simulated night work. Chronobiol. Int. 35, 229-239. https://doi.org/10.1080/07420528.2017.1394321 (2018).

34. Sadeghniiat-Haghighi, K., Yazdi, Z., Jahanihashemi, H. \& Aminian, O. The effect of bright light on sleepiness among rapid-rotating 12-hour shift workers. Scand. J. Work Environ. Health 37, 77-79 (2011).

35. Sunde, E. et al. Role of nocturnal light intensity on adaptation to three consecutive night shifts: A counterbalanced crossover study. Occup. Environ. Med. 77, 249. https://doi.org/10.1136/oemed-2019-106049 (2020).

36. Tanaka, K. et al. Brief morning exposure to bright light improves subjective symptoms and performance in nurses with rapidly rotating shifts. J. Occup. Health 53, 258-266. https://doi.org/10.1539/joh.L10118 (2011).

37. Thorne, H. C., Hampton, S. M., Morgan, L. M., Skene, D. J. \& Arendt, J. Returning from night shift to day life: Beneficial effects of light on sleep. Sleep Biol. Rhythms 8, 212-221 (2010).

38. Yoon, I. Y., Jeong, D. U., Kwon, K. B., Kang, S. B. \& Song, B. G. Bright light exposure at night and light attenuation in the morning improve adaptation of night shift workers. Sleep 25, 351-356 (2002).

39. Harma, M., Sallinen, M., Ranta, R., Mutanen, P. \& Muller, K. The effect of an irregular shift system on sleepiness at work in train drivers and railway traffic controllers. J. Sleep Res. 11, 141-151 (2002).

\section{Acknowledgements}

The authors thank Mr. Zero Chan, Ms. Henny Dwi Susanti, and Ms. Yi-Hsuan Hsu for their assistance on research and administrative work, and also thank Wallace Academic Editing for editing the paper for English grammar and usage.

\section{Author contributions}

C.L. contributed to the data collection and analysis, interpretation, and drafting of the manuscript. M.H.C. contributed to the data analysis, interpretation, and drafting of the manuscript.

\section{Competing interests}

The authors declare no competing interests.

\section{Additional information}

Correspondence and requests for materials should be addressed to M.-H.C.

Reprints and permissions information is available at www.nature.com/reprints.

Publisher's note Springer Nature remains neutral with regard to jurisdictional claims in published maps and institutional affiliations. 
(c) (i) Open Access This article is licensed under a Creative Commons Attribution 4.0 International cc) License, which permits use, sharing, adaptation, distribution and reproduction in any medium or format, as long as you give appropriate credit to the original author(s) and the source, provide a link to the Creative Commons licence, and indicate if changes were made. The images or other third party material in this article are included in the article's Creative Commons licence, unless indicated otherwise in a credit line to the material. If material is not included in the article's Creative Commons licence and your intended use is not permitted by statutory regulation or exceeds the permitted use, you will need to obtain permission directly from the copyright holder. To view a copy of this licence, visit http://creativecommons.org/licenses/by/4.0/.

(C) The Author(s) 2021 